\title{
Selective Temporary Stent-Assisted Coil Embolization for Intracranial Wide-Necked Small Aneurysms Using Soli- taire AB Retrievable Stent
}

\author{
Han Yong Heo, M.D., Jae Guen Ahn, M.D., ${ }^{1}$ Cheol Ji, M.D., Ph.D., ${ }^{1}$ Won Ki Yoon, M.D., Ph.D. ${ }^{2}$ \\ Department of Neurosurgery, 'St. Paul's Hospital, College of Medicine, The Catholic University of Korea, Seoul, Korea \\ Department of Neurosurgery, ${ }^{2}$ Korea University Guro Hospital, Korea University College of Medicine, Seoul, Korea
}

Objective : Stent-assisted coil embolization of intracranial wide-necked aneurysm requires long-term postoperative antiplatelet therapy to prevent in-stent thrombosis. This study aimed to demonstrate results of temporary stent placement for coiling wide necked small intracranial aneurysms, which eliminated need for antiplatelet agents, and to discuss its feasibility and safety.

Methods : Data of 156 patients who underwent stent-assisted coil embolization between 2011 and 2014 were retrospectively analyzed. Thirteen cases of temporary stent-assisted coil embolization were included, and their clinical and radiological results were evaluated.

Results : The aneurysms treated were all unruptured except one case. All of them had wide neck with mean dome-to-neck ratio of 0.96 and were small-sized aneurysms with mean maximal diameter of $4.2 \mathrm{~mm}$. There was no technical failure in retrieval of stent after completion of embolization of the target aneurysm. Immediate angiography revealed 11 complete and two partial embolization (one residual neck and one residual aneurysm). Two cases encountered thrombosis complication, and they were managed without neurological sequelae. The mean follow-up period was 43 months, angiographic follow-up revealed two cases with minor recurrence, and clinical outcome was good with modified Rankin scale score of 0 .

Conclusion : Temporary stent-assisted coil embolization of small wide-necked intracranial aneurysm using fully retrievable stent appears safe and effective. Further application and evaluation of this technique in more cases with larger size aneurysm is warranted.

Key Words : Coil · Intracranial aneurysm · Stents.

\section{INTRODUCTION}

Endovascular treatment of intracranial aneurysms is a wellestablished treatment option for majority of patients ${ }^{13)}$. Treat- ment of wide-necked aneurysm is challenging and requires various endovascular techniques, such as neck remodeling with stent or balloon, multi-catheter technique, neck bridging with Comaneci (Rapid Medical, Yokneam, Israel) or Woven

- Received : March 20, 2018 •Revised : August 9, 2018 •Accepted : October 12, 2018

- Address for reprints : Won Ki Yoon, M.D., Ph.D.

Department of Neurosurgery, Korea University Guro Hospital, Korea University College of Medicine, 148 Gurodong-ro, Guro-ru, Seoul 08308, Korea Tel : +82-2-2626-1178, Fax : +82-2-863-1684, E-mail : nvkumc@gmail.com

This is an Open Access article distributed under the terms of the Creative Commons Attribution Non-Commercial License (http://creativecommons.org/licenses/by-nc/4.0) which permits unrestricted non-commercial use, distribution, and reproduction in any medium, provided the original work is properly cited. 
EndoBridge (Sequent Medical, Aliso Viejo, CA, USA) device ${ }^{7)}$. Specifically, the stent-assisted coil embolization technique is commonly used ${ }^{3}$. However, after deployment of the stent for neck remodeling, antiplatelet agents should be initiated and continued, which can be beneficial for prevention of ischemic stroke but also can be a risk factor for hemorrhagic complication $^{3,5}$. To reduce the chance of using antiplatelet agents after the procedure, balloon-assisted coil embolization would be an alternative. However, temporary flow arrest during balloon inflation can induce ischemic insult and affect brain function. Sometimes, we encounter some serious complications such as rupture of the aneurysm or parent artery caused by the wall pressure of the aneurysm or parent artery ${ }^{4}$. Considering the risk and benefit in treating the small aneurysms enables us to treat them using simple and less risky methods. For this purpose, we employed temporary stent-assisted coil embolization of intracranial aneurysms using fully retrievable and detachable Solitaire AB stent in selected cases. Here, we describe the technique and clinical and radiological results and discuss the feasibility of this technique.

\section{MATERIALS AND METHODS}

After obtaining approval from Institutional Review Board of St. Paul's Hospital (IRB No. Pc17Resi0075), we retrospectively searched for all the medical records of patients whose aneurysms were treated with the stent-assisted technique from January 2011 to January 2015. During this time period, we treated 156 cases of aneurysm with the stent-assisted technique. From March 2011 to June 2013, we treated 13 cases of small intracranial aneurysms with temporary stent-assisted coil embolization technique (TemSAT) and followed them. TemSAT is indicated for saccular, wide-necked, small intracranial aneurysms. The exclusion criteria were aneurysms with very low aspect ratio, which causes the funnel shape of the aneurysm; extremely wide neck encompassing half of the circumference of the parent artery; and large aneurysms with diameter over $10 \mathrm{~mm}$. Patient demographic data are shown in Table 1. Analysis and evaluation were focused on the possibility of stent recapture after coiling, occlusion rate, recurrence of aneurysm, and procedure-related complication rate. Aneurysm occlusion was determined by using the modified Raymond-Roy classification ${ }^{11)}$. Clinical pre- and post-interven- tional status of each patient was graded according to the modified Rankin scale (mRS).

\section{Endovascular procedure}

Doses of $100 \mathrm{mg}$ of acetyl salicylic acid and $75 \mathrm{mg}$ of clopidogrel were administered orally at least 7 days prior to the procedure for unruptured aneurysms. For ruptured aneurysms, preoperative antiplatelet agents were not used. All procedures were performed via the transfemoral access route under general anesthesia. Heparin sulfate was injected intravenously to maintain 2 to 2.5 times of the baseline activated clotting time for unruptured aneurysms. For ruptured cases, 3000 IU of intravenous heparin was injected after the second coil was deployed. A large-bore (over 6 French [Fr]) guiding catheter was placed in the cervical internal carotid artery (ICA), and a microcatheter was introduced into the aneurysm sac. For stent delivery, a Prowler select plus microcatheter (Codman Neurovascular, Raynham, MA, USA) was inserted into the parent artery beyond the lesion.

Coiling was initiated without deployment of the neck remodeling stent. After attempting to frame the first coil within the aneurysm, stent deployment was determined according to the stability of the frame. If the first framing coil protruded to the parent artery or appeared unstable during deployment, a Solitaire AB stent (Covidien, Irvine, CA, USA) was inserted and deployed to cover the aneurysm neck. Subsequent coiling was performed until complete obliteration of the aneurysm was confirmed. After slight withdrawal of the coiling microcatheter and keeping it just proximal to the coil mass still jailed by the stent and the vessel wall, the deployed stent was recaptured. By pushing the stent delivery catheter and pulling the stent wire, the stent was recaptured without moving it. Once the coil mass was stable after partial recapture of the stent, thereby exposing the aneurysm neck, the coiling catheter was removed completely. This maneuver confirmed whether the coil mass was stable or not. The stent was recovered, and the delivery catheter was removed completely after final confirmation of the stability of the coil mass. Rotational angiography was performed to check any intracranial vascular complication. The femoral artery access site was sealed, and hemostasis was completed. 


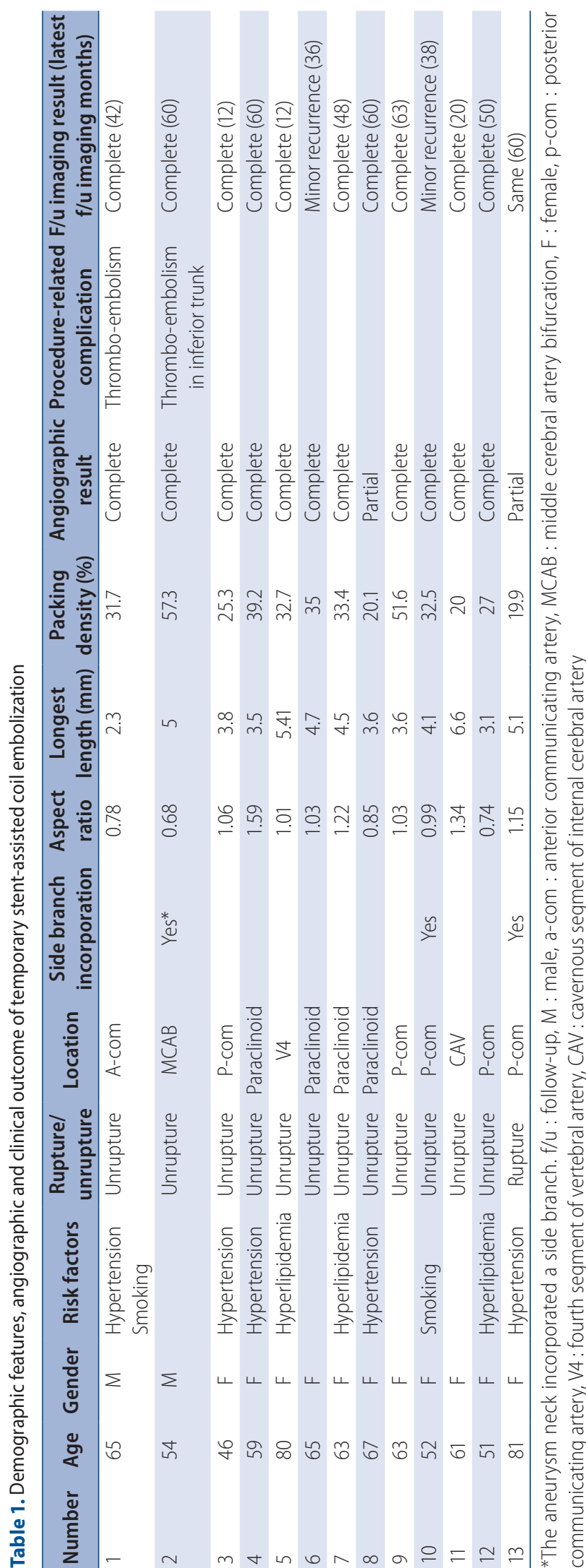

\section{RESULTS}

The aneurysms were all unruptured except for one case of subarachnoid hemorrhage (SAH). The posterior communicating artery aneurysm was the most frequent with five cases, followed by the paraclinoid aneurysm with four cases. All aneurysms were wide necked with aspect ratio below 1.5 (mean, 0.96 ; range, 0.63 to 1.34 ). The mean size of the aneurysms was $4.2 \mathrm{~mm}(2.3-6.6 \mathrm{~mm})$. They were all treated with TemSAT. All cases that were indicated for this technique were successfully treated. Any failure to recapture the stent due to wedged coil in the stent or movement of the coil mass after retrieval of the stent did not occur.

The packing density by $3 \mathrm{D}$ volumetric calculation with the angiography workstation was $32.75 \%$. The immediate angiographic results were as follows : 11 complete $(84.6 \%)$ and two partial embolizations (residual neck as class II and residual aneurysm as class IIIa, respectively) (Fig. 1) ${ }^{11)}$. Periprocedural complications with thromboembolism were encountered in two cases, which were treated successfully without any neurological sequelae. All cases were followed up clinically and radiologically for at least 1 year (range, 12 to 63 months; mean, 43 months). The clinical outcome was excellent without any mortality or morbidity (mRS 0). Angiographic follow-up results showed two cases of minor recurrence. The latter two cases with partial embolization were found to be improved and stable.

\section{Illustrated case}

A 63-year-old woman, whose unruptured paraclinoid ICA aneurysm was incidentally found, visited our institute. Angiography revealed a wide-necked small aneurysm with aspect ratio of 1.22 and height of $4.5 \mathrm{~mm}$. A 6-Fr guiding catheter was placed in the cervical ICA, and a steam shaped as a pig tail to the right side of the Excelsior SL-10 (Stryker, Fremont, CA, USA) microcatheter was introduced into the aneurysm sac. A target soft $3604.0 \mathrm{~mm}$ by $6 \mathrm{~cm}$ coil (Stryker) was deployed into the aneurysm for framing. However, the coil delivery catheter appeared unstable to escape from the aneurysm. To stabilize the microcatheter, a Solitaire AB stent of $5 \times 20 \mathrm{~mm}$ was deployed, covering the neck of the aneurysm using Prowler select plus 45 degree preshaped microcatheter (Codman Neurovascular). Subsequently, four more coils were packed into the aneurysm safely, isolating the aneurysm out of 
the blood flow. Before withdrawal of the aneurysm microcatheter, the stent-delivery microcatheter was re-introduced slowly to recapture the stent. During this recapture, the coil mass did not show any movement or instability. The microcatheter harboring the stent was left in-situ before the aneurysm microcatheter was withdrawn gently and removed safely without any change in the coil mass. Then, the stent-delivery microcatheter was removed. Final angiography demonstrated complete coil embolization of the aneurysm without any complication. The patient recovered without any neurological deficit and was discharged the next day. Her 4-year follow-up angiogram showed stable coil without recurrence (Fig. 2).

\section{DISCUSSION}

With technological evolution at present, endovascular treatment of intracranial aneurysm has become popular and easier than in the past. As revealed in the International Subarachnoid Aneurysm Trial, endovascular management is a safe modality for the treatment of intracranial aneurysms ${ }^{13}$. For widenecked aneurysms, various techniques and materials have been developed in the endovascular arena. Specifically, given the ease of deployment, maintenance of the parent artery patency during procedure, and future endothelialization that helps in the externalization of the aneurysm from the blood circulation, stents are widely used and become more popular than the balloon-assisted technique $e^{2,3,8,10,12}$. However, it harbors some drawbacks : risks of periprocedural in-stent thromboembolism, in-stent stenosis by excessive endothelialization,
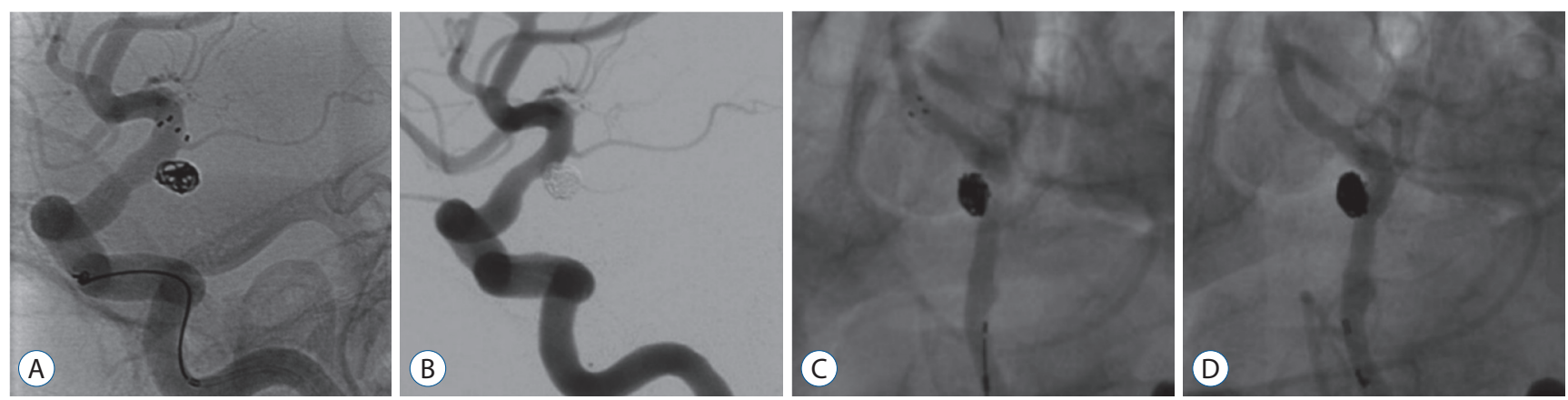

Fig. 1. Examples of immediate complete embolization with temporary stent-assisted coil embolization. A and B : A case of posterior communicating artery unruptured aneurysm. A : Stent-assisted coil embolization is performed with jail technique on a posterior communicating artery unruptured aneurysm with wide neck. B : Immediate post-procedure angiography shows completely embolized aneurysm with removed stent without any complicated features. C and D : A case of vertebral artery unruptured aneurysm. C : An unruptured left vertebral artery wide-necked aneurysm is treated with jailed stent-assisted technique. The stent is fully deployed. D : After removal of the stent, the aneurysm is completely embolized and the parent artery is patent.
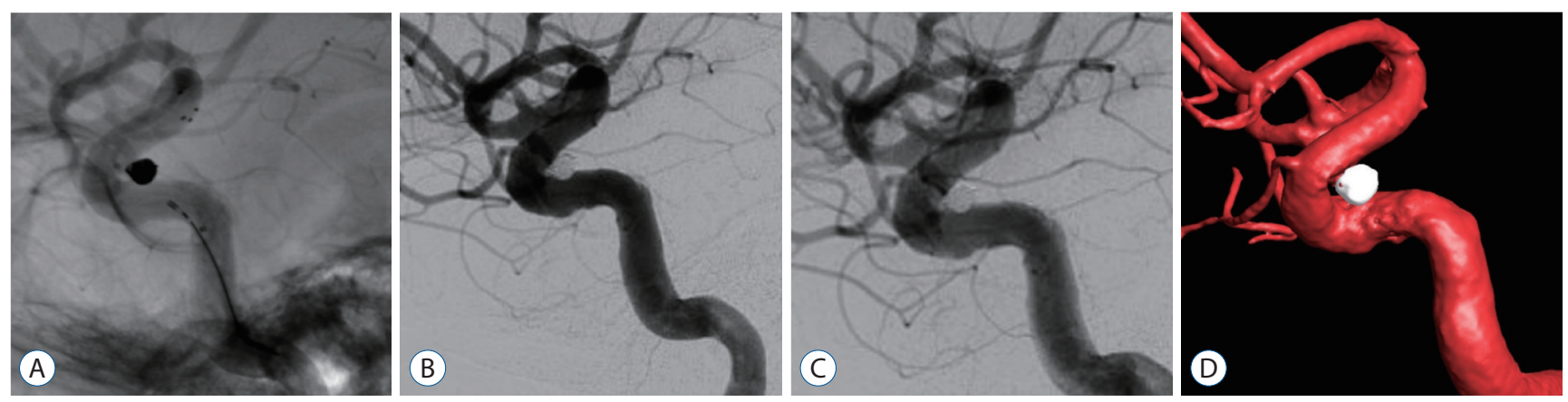

Fig. 2. Illustrated case of a paraclinoid aneurysm. A : To stabilize the catheter, a Solitaire $A B 5 \times 20 \mathrm{~mm}$ stent is deployed covering the neck of the aneurysm. Subsequent coil deployment is performed easily. B : After completion of coil packing in the aneurysm, the stent is recaptured and removed. $C$ : First angiographic follow-up at 2 years shows complete and stable packing of the aneurysm. D : Second angiographic follow-up at 4 years demonstrates complete exclusion of the aneurysm out of the blood circulation. 
and the need for life-long antiplatelet agent administration ${ }^{3,5,8,15}$. Considering the pursuit of minimalism and less invasiveness of procedure and efforts to reduce periprocedural complication, permanent deployment of the stent appears excessive, especially when treating small unruptured aneurysms with wide neck. This is our reason of employing the TempSAT. For ruptured cases, we used this technique in case 13 to reduce risk of periprocedural complication induced by permanent placement of stent during the acute hemorrhagic phase. No specific technical feature is available in this particular case. Müller et al. ${ }^{14)}$ reported their experience of this technique with 20 cases of ruptured aneurysms. They pointed out the merit that platelet inhibition is not needed after the procedure on patients with $\mathrm{SAH}^{14)}$.

All procedures were performed with technical success. Stent retrieval was easy and safe, and the coil mass did not move at all. Coil engagement into the stent did not occur. The packing density and completeness of coil embolization was comparable to typical stent-assisted coil embolization ${ }^{3,8,10,12)}$. Temporary neck remodeling was successfully performed with TemSAT, with the following advantages. First, in the stent-assisted technique, the patency of the parent artery was maintained throughout the procedure. Unlike the balloon-assisted technique, stents do not block blood flow during the procedure, which reduced possible ischemic insult to the affected brain $^{3,4,8}$. Second, TemSAT stabilized the microcatheter and enhanced coil stabilization ${ }^{3,8,12)}$. Because of the wide neck of the aneurysm or geometrical relationship of the aneurysm and parent artery, the catheter or coil mass would become unstable during embolization. However, the stent stabilized the microcatheter by abutting it to the vessel wall and prevented the so-called kick-back phenomenon. Moreover, the saccular, wide-necked aneurysm could be coiled safely because the stent kept the coil in the aneurysm until it could become stable by subsequent packing. And third, any antiplatelet agents were not necessary after the procedure to reduce risk of future in-stent thromboembolism or stenosis. Because the stent was removed from the cerebral artery by recapturing, the stent itself did not increase the risk of thromboembolism after the procedure. This was helpful in ruptured case. If further surgical procedures such as extraventricular drainage were anticipated, antiplatelet agents would become a very potential risk factor for hemorrhagic complication ${ }^{1,15,16)}$.

However, this technique showed some drawbacks as well.
Technically, if a coil loop became lodged in the strut of the stent, it cannot be recaptured and removed. It should be left as it is. By using the jail technique, this risk can be reduced, but it is not eliminated completely. Hence, it is crucial to confirm that any coil loop is not engaged in the stent strut.

Stent is known to reduce aneurysm recurrence rate by instent endothelialization, possible flow diversion effect, and eventual isolation of the aneurysm from the circulation ${ }^{17,18)}$. TemSAT does not provide this theoretical chance. However, with this low profile and low metal-covered stent, flow diversion effect or rapid and full endothelialization is not expected very positively. In spite of this hypothetical drawback, 11 of 13 $(85 \%)$ cases showed stable features in relatively long-term follow-up angiography.

Furthermore, this technique may not be applied on every wide-necked aneurysm. If the aneurysm has very low aspect ratio, wide with low funnel shape, or very wide neck encompassing almost half the circumference of the parent artery, using the usual or combined stent-assisted coil embolization technique is advisable ${ }^{2,6}$.

We applied this technique only to small aneurysms, and its immediate and follow-up radiological outcomes were excellent. Given that there are only several reports with small numbers of cases on this technique ${ }^{1,7,14,16,19)}$, we cannot conclude that this technique could be used on any size of aneurysms. Fischer et al. ${ }^{7)}$ described their success in 18 cases of temporary neck bridging with Comaneci device, and the mean size of the aneurysm was $6.0 \mathrm{~mm}(2.0-12 \mathrm{~mm})$. The largest case series described by Müller et al. ${ }^{14)}$ demonstrated small to large aneurysms with mean maximal size of $7 \mathrm{~mm}$. Signorelli et al. ${ }^{16)}$ reported eight cases of treatment with this technique on ruptured aneurysms with various and relatively larger sizes than the previous studies (mean, $10.8 \mathrm{~mm}$; range, 3 to $22 \mathrm{~mm}$ ). However, the clinical and radiological follow-up period was very short on these eight patients, so recurrence was not evaluated in their study ${ }^{16)}$. Considering the angiographic results of the current study and other studies, aneurysm of any size can be treated using this technique with excellent immediate results, but the durability on large-sized aneurysm should be left open for discussion and research.

The complications encountered in this case series were two cases of thromboembolism. In those cases, the stents were removed, and subsequent chemical thrombolysis achieved full recanalization without any neurological sequelae. In case 1 
(Fig. 3A-D), during coiling of the anterior communicating artery aneurysm after stent deployment in the parent artery, flow limitation with wrinkling of proximal A1 and geometric angle change from the ICA to the anterior cerebral artery occurred, and eventual in-stent thrombosis developed ${ }^{9)}$. Then, 50000 IU of intra-arterial urokinase was administered, but it failed to recanalize the occluded stent. The stent was removed after complete packing of the aneurysm, and the thrombus dissolved. Postoperative diffusion magnetic resonance imaging did not show any meaningful signal restriction. In case 2 (Fig. 3E-H), the inferior trunk of the middle cerebral artery bifurcation was occluded during the procedure. Finally, after confirming the stability of the coil mass, the stent was recaptured and removed. And subsequent intra-arterial chemical thrombolysis using $160000 \mathrm{IU}$ of urokinase and $2.375 \mathrm{mg}$ of tirofiban successfully lysed the thrombus in the inferior trunk. This patient did not show any neurological deficit.
These two complications did not leave any sequelae during the follow-up period. And they occurred due to the stent assistance itself not the stent retrieval.

For safety concern, we cautiously suggest indication and contraindication on the retrieval of stent. This technique might be applied on the cases with relatively wide-necked small saccular aneurysm without funnel shape, using a fully retrievable stent. A funnel shaped or too low height widenecked aneurysm, suspicion of coil loop engagement on the stent, any slight movement of coil mass during recapture, poor pathological condition in the stented segment parent artery or technical difficulty that hinders recapture of stent without toand-fro movement of stent should be considered as contraindication of this technique. And as shown on our cases of thromboembolic complication, this technique should be applied with caution in the cases of distal aneurysm with small caliber parent artery.
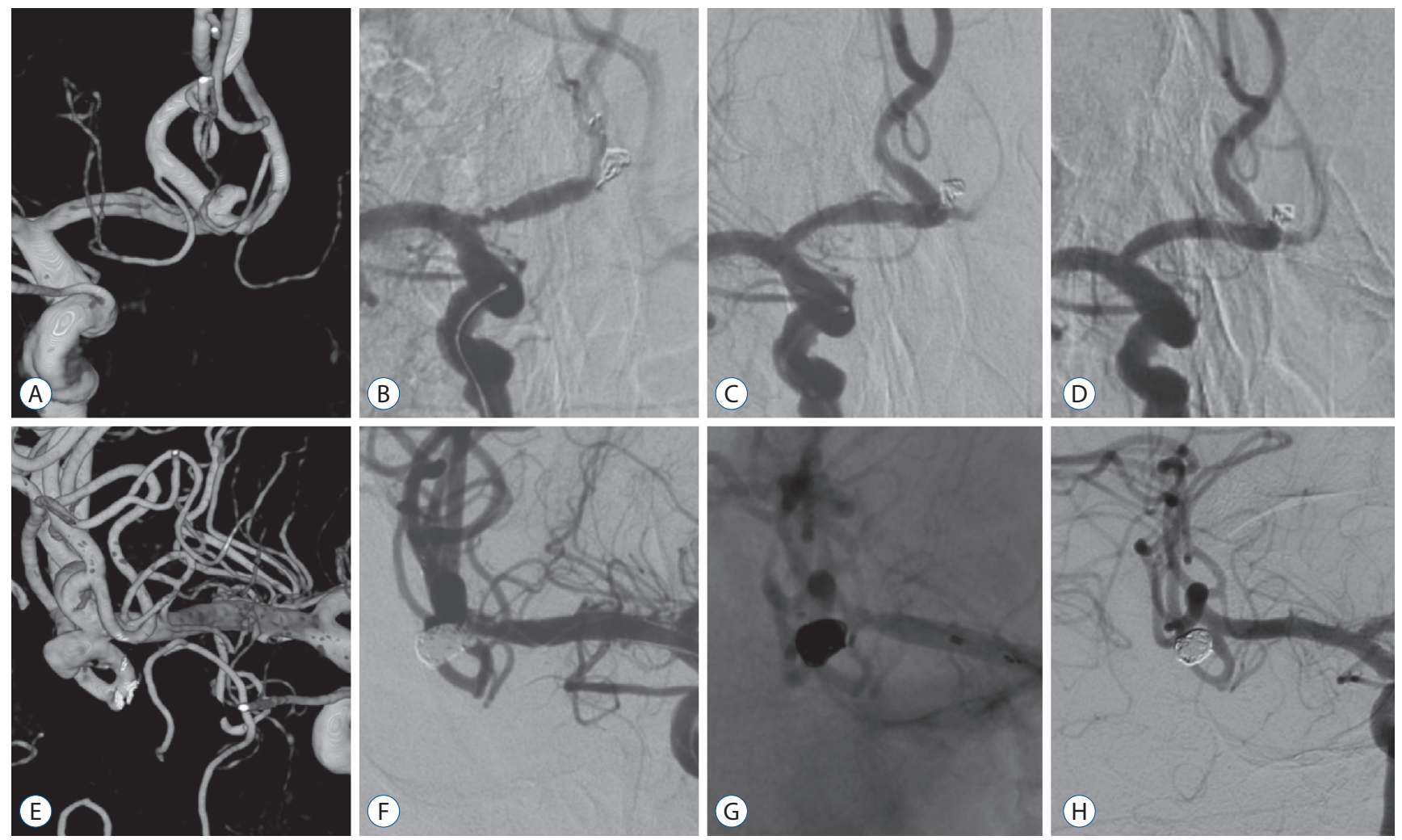

Fig. 3. Cases of thromboembolic complication. A-D : A case of anterior communicating artery aneurysm. A : The anterior communicating artery aneurysm has a wide neck. B : Change in vascular geometry and wrinkling of proximal A1 segment with distal flow stagnation by stent deployment is observed. The angle between A1 and A2 is changed compared to that in Fig. 3A. C: Note the restored vascular geometry and patent arteries after stent removal. D : Follow-up angiography at 3 years shows stable and complete embolization and patent parent arteries. E-H : A case of middle cerebral artery aneurysm. E : A wide-necked middle cerebral artery bifurcation aneurysm incorporating inferior trunk. F : At the final stage of stent-assisted coil embolization of the aneurysm, thrombosis occurrs in the origin of inferior trunk. $G$ : The stent is removed safely and chemical thrombolysis is performed to achieve full recanalization. $\mathrm{H}$ : Follow-up angiogram at 5 years shows completely embolized aneurysm with patent inferior trunk. 


\section{Limitation of the current study}

The nature of the retrospective study gives a big selection bias. The small number of cases limits the universal application of this technique to all intracranial aneurysms. Specifically for SAH, we implied this technique in one case only. Even though there are previous reports about the application of this technique on ruptured aneurysms, we cannot conclude that this technique can be safely introduced to the ruptured cases.

Although there are several reports about this technique, the number of cases is very small ${ }^{1,7,14,16,19)}$. The largest one is that by Müller et al. ${ }^{14)}$ with 33 cases. We believe that this technique is valuable to be researched with safe indications.

\section{CONCLUSION}

TemSAT was used successfully in several cases of widenecked small aneurysms. Its clinical and radiologic follow-up results were acceptable in mid-term period. Thromboembolic complications were treated successfully by stent removal and chemical thrombolysis. We discussed the advantages and disadvantages of this technique in terms of safety, and this provides impression of the feasibility and safety of this technique on selected small wide-necked saccular aneurysm.

\section{CONFLICTS OF INTEREST}

No potential conflict of interest relevant to this article was reported.

\section{INFORMED CONSENT}

This type of study does not require informed consent.

\section{References}

1. Almekhlafi MA, Hockley A, Wong JH, Goyal M : Temporary Solitaire stent neck remodeling in the coiling of ruptured aneurysms. J Neurointerv Surg 5 Suppl 3 : iii76- iii78, 2013

2. Brinjikji W, Cloft HJ, Kallmes DF : Difficult aneurysms for endovascular treatment: overwide or undertall? AJNR Am J Neuroradiol 30 : 1513-
1517, 2009

3. Chalouhi N, Jabbour P, Singhal S, Drueding R, Starke RM, Dalyai RT, et al. : Stent-assisted coiling of intracranial aneurysms: predictors of complications, recanalization, and outcome in 508 cases. Stroke 44 : 1348-1353, 2013

4. Chalouhi N, Jabbour P, Tjoumakaris S, Dumont AS, Chitale R, Rosenwasser $\mathrm{RH}$, et al. : Single-center experience with balloon-assisted coil embolization of intracranial aneurysms: safety, efficacy and indications. Clin Neurol Neurosurg $115: 607-613,2013$

5. Choi HH, Lee JJ, Cho YD, Han MH, Cho WS, Kim JE, et al. : Antiplatelet premedication for stent-assisted coil embolization of intracranial aneurysms: low-dose prasugrel vs clopidogrel. Neurosurgery 83 : 981 988, 2018

6. Fiorella D, Albuquerque FC, Masaryk TJ, Rasmussen PA, McDougall CG : Balloon-in-stent technique for the constructive endovascular treatment of "ultra-wide necked" circumferential aneurysms. Neurosurgery 57 : 1218-1227; discussion 1218-1227, 2005

7. Fischer S, Weber A, Carolus A, Drescher F, Götz F, Weber W : Coiling of wide-necked carotid artery aneurysms assisted by a temporary bridging device (Comaneci): preliminary experience. J Neurointerv Surg 9 : 1039-1097, 2017

8. Hetts SW, Turk A, English JD, Dowd CF, Mocco J, Prestigiacomo C, et al. : Stent-assisted coiling versus coiling alone in unruptured intracranial aneurysms in the matrix and platinum science trial: safety, efficacy, and mid-term outcomes. AJNR Am J Neuroradiol 35 : 698-705, 2014

9. Huang QH, Wu YF, Xu Y, Hong B, Zhang L, Liu JM : Vascular geometry change because of endovascular stent placement for anterior communicating artery aneurysms. AJNR Am J Neuroradiol 32 : 1721-1725, 2011

10. Jeong HW, Seung WB : Outcomes of stent-assisted coil embolization of wide-necked intracranial aneurysms using the Solitaire ${ }^{\mathrm{TM}} \mathrm{AB}$ neurovascular remodeling device. J Cerebrovasc Endovasc Neurosurg 17 : 301-312, 2015

11. Mascitelli JR, Moyle H, Oermann EK, Polykarpou MF, Patel AA, Doshi $\mathrm{AH}$, et al. : An update to the Raymond-Roy Occlusion Classification of intracranial aneurysms treated with coil embolization. J Neurointerv Surg 7 : 496-502, 2015

12. Mocco J, Snyder KV, Albuquerque FC, Bendok BR, Alan S B, Carpenter JS, et al. : Treatment of intracranial aneurysms with the Enterprise stent: a multicenter registry. J Neurosurg 110:35-39, 2009

13. Molyneux AJ, Birks J, Clarke A, Sneade M, Kerr RS : The durability of endovascular coiling versus neurosurgical clipping of ruptured cerebral aneurysms: 18 year follow-up of the UK cohort of the International Subarachnoid Aneurysm Trial (ISAT). Lancet 385 : 691-697, 2015

14. Müller M, Brockmann C, Afat S, Nikoubashman O, Schubert GA, Reich A, et al. : Temporary stent-assisted coil embolization as a treatment option for wide-neck aneurysms. AJNR Am J Neuroradiol 38 : 1372-1376, 2017

15. Ryu CW, Park S, Shin HS, Koh JS : Complications in stent-assisted endovascular therapy of ruptured intracranial aneurysms and relevance to antiplatelet administration: a systematic review. AJNR Am J Neurora- 
diol 36 : 1682-1688, 2015

16. Signorelli F, Gory B, Turjman F : Temporary solitaire stent-assisted coiling: a technique for the treatment of acutely ruptured wide-neck intracranial aneurysms. AJNR Am J Neuroradiol 35 : 984-988, 2014

17. Young JJ : Neointimal formation following drug-eluting stents: physiology, timeline, and the influence of drug delivery systems. Rev Cardiovasc Med 8 Suppl 1 : S3-S10, 2007
18. Zhu W, Tian Y, Zhou LF, Wang Y, Song D, Mao Y, et al. : Development of a novel endothelial cell-seeded endovascular stent for intracranial aneurysm therapy. J Biomed Mater Res A 85 : 715-721, 2008

19. Zumofen DW, Sahasrabudhe N, Riina HA, Raz E, Shapiro M, Becske T, et al. : Temporary stent scaffolding during aneurysm coiling. J Clin Neurosci $21: 852-854,2014$ 